\title{
Molecular Simulation of Phase Coexistence in Adsorption in Porous Solids
}

\author{
MIGUEL JORGE and NIGEL A. SEATON* \\ School of Chemical Engineering, University of Edinburgh, King's Buildings, \\ Mayfield Road, Edinburgh, EH9 3JL, UK \\ *Corresponding Author \\ Tel.: (+44) 131650 4867; Fax: (+44) 1316506551 \\ e-mail: nigel@chemeng.ed.ac.uk
}




\begin{abstract}
In this work a recently proposed method, the gauge-cell Gibbs ensemble Monte Carlo, is extended to deal with polar substances. The behaviour of water, a hydrogen bonding, weakly adsorbing fluid, is compared with that of methane, a strongly adsorbing, non-polar fluid, in the vicinity of the phase transition. The mechanisms of condensation for the two species are seen to be significantly different in nature. A systematic study of the effect of the pore width on the phase equilibrium of water is also performed. Our results show that the narrowing of the pore shifts the equilibrium transition pressure to lower values and reduces the extent of vapour metastability, but exerts little influence on the stability of the liquid phase.
\end{abstract}

\title{
1 Introduction
}

First-order phase transitions occur widely in adsorption in porous materials. Vapour-liquid equilibrium in pores occurs in several systems of interest, such as adsorption of $\mathrm{N}_{2}$ in mesoporous solids and water in both microporous and mesoporous solids [1]. The term "vapour" is used here by analogy with bulk vapour-liquid transitions; in the context of adsorption, the "vapour phase" corresponds to a situation in which some molecules are adsorbed on the pore walls, with others in gas-like configurations in the inner part of the pore. The attractive interaction between the pore walls and the adsorbate molecules causes the equilibrium transition pressure (at constant temperature) to shift to lower values - capillary condensation [2]. A critical pore width also exists, below which the transition is continuous and reversible $[3,4]$. Throughout the years, there have been numerous attempts to describe vapour-liquid equilibrium in porous solids, both experimentally and theoretically (for a recent review, see [5]). Experimental studies in relatively uniform and well characterised 
adsorbents, such as MCM-41 and controlled porous glasses, have been recently reported (see [5] and references therein). Such measurements, however, can be made only for a limited class of materials, not including, for example, activated carbons. Molecular simulation, on the other hand, allows one to study phase equilibrium in individual pores as well as to assess the influence of structure and chemistry of the adsorbent on the phase coexistence. This is particularly useful in cases where the underlying mechanisms of adsorption and phase equilibrium are not entirely understood. The adsorption of water on activated carbon is one such case.

So far, most molecular simulation studies of vapour-liquid coexistence in confined media have focused on simple fluids (usually described by the LennardJones potential) adsorbed in smooth slit-shaped or cylindrical pores. There have been few published studies in which vapour-liquid equilibrium of confined water was examined. Spohr et al. [6] observed the occurrence of capillary condensation in a molecular dynamics simulation of water between planar walls. Unfortunately, neither the pressure nor the chemical potential were calculated in the course of the simulation, so comparisons with experiments are not possible. Luzar et al. [7] have used a latticegas model to study the dynamics of capillary evaporation of water between hard walls. They found that the presence of hydrophilic sites on the surface exerted a strong influence on the mechanism of phase transition. A series of papers have been published by Gubbins and co-workers [8-11] on the subject of molecular simulation of water in carbon pores. The authors have provided insight on the structure of adsorbed water [8], as well as on the mechanism of water adsorption $[9,11]$. While this work provided evidence for the occurrence of capillary condensation in carbon pores, the grand canonical Monte Carlo method used by the authors is (as we will see later) incapable of directly simulating phase equilibrium. To our knowledge, only two 
papers have been published in which phase equilibrium data for confined water has been determined. Brodskaya et al. [12] calculated the chemical potential at the equilibrium transition for water between hard walls, while Brovchenko et al. [13] presented vapour-liquid coexistence curves in smooth cylindrical pores for several strengths of the solid-fluid interaction. In this paper we present for the first time results from molecular simulations of water in slit-shaped graphitic pores at the equilibrium phase transition. The behaviour of water is compared to that of a simple, non-polar fluid - in this case, methane. We also present an analysis of the effect of pore width on the coexistence properties.

The paper is organised as follows. In section 2 we review several methods for the simulation of phase equilibrium in confined media, while discussing their applicability to the simulation of water in activated carbon pores. Section 3 describes the potential models employed for the simulation of the adsorption of water and methane in slit-shaped graphitic pores (representing the pores in an activated carbon adsorbent) and presents technical details of the simulation methods used. In section 4 we present the results and contrast the condensation behaviour of water and methane. Finally, we make some conclusions in section 5.

\section{Methods for Simulation of Phase Equilibrium}

Most molecular simulations of adsorption studies are performed in the grand canonical ensemble $[14,15]$. In this type of simulation, the temperature $(T)$, volume $(V)$ and chemical potential $(\mu)$ are kept constant, while the total number of molecules

$(N)$ is allowed to fluctuate. By calculating the average density at the end of each run, it is possible to plot equilibrium adsorption isotherms for pores of different sizes and characteristics. However, when calculating isotherms by grand canonical Monte Carlo (GCMC) simulation in the vicinity of a phase transition, the results exhibit strong 
hysteresis. figure 1 shows a sketch of a typical isotherm for the case of a vapourliquid transition. When approaching the phase transition from low pressures, as in a typical adsorption experiment (line OCHI), the vapour branch overshoots the equilibrium condensation pressure and remains metastable until reaching point $\mathbf{C}$, when it spontaneously condenses into the liquid state. Similarly, if we lower the pressure starting from a liquid configuration, as in a desorption experiment (line IFAO), we observe metastable liquid states below the equilibrium condensation pressure, up to the point of spontaneous evaporation (point F). The theoretical isotherm for this type of system would exhibit a van der Waals loop (line ODEI), in which points $\mathbf{D}$ and $\mathbf{E}$ represent the limits of stability of the vapour and liquid phases, respectively (the spinodal points). Between these two points the fluid can be found in either the vapour or the liquid state, with one of these phases being metastable with respect to the other. Beyond these points, however, only one stable phase exists. Spontaneous condensation and evaporation occur when the density fluctuations inside the pore are large enough to allow the free energy barrier that separates the phases to be overcome, always before the spinodal limits of stability. In principle, if we were to allow an infinite number of MC steps, the system would always overcome the free energy barrier and reach the most stable phase. Thus, the true equilibrium phase transition (segment BG) would be attainable [16]. In practice, however, hysteresis is unavoidable in GCMC simulations. [Insert figure 1 about here]

Since the free energy of the system is not an output of the GCMC simulation, it is not possible to directly calculate the conditions of phase equilibrium. A way to circumvent this problem was proposed by Peterson and Gubbins [17]. In this method, one must construct two reversible paths from a reference state for which the free energy is known (or can be calculated) up to the metastable vapour and liquid 
branches. The free energy can then be calculated by integrating along each of those paths, and the condition of phase equilibrium is satisfied at the point where the free energies are equal. The reference state is usually the ideal gas, from which the metastable vapour phase is easily accessible. However, to access the metastable liquid phase, one must produce a reversible supercritical isotherm, as well as a curve at constant chemical potential to link both the supercritical and the subcritical isotherms. The effort involved in these calculations is quite large, particularly in the case of polar substances like water, where the interactions are long-ranged. Additionally, the integration along the path of constant chemical potential seems to present accuracy problems [18].

An elegant method that avoids the need for thermodynamic integration is the Gibbs ensemble Monte Carlo (GEMC) of Panagiotopoulos, originally proposed for the simulation of bulk coexistence [19], and later extended to confined systems [20]. The advantage of this procedure is that it is able to simulate both the liquid and vapour phases without the need for an explicit interface. The system consists of two separate simulation cells at the same temperature, but with the possibility of exchanging molecules and volume between them (the total $N$ and $V$ remaining constant). After equilibration, one of the pores will contain liquid, while the other will contain vapour (the coexisting densities are, therefore, easily obtainable). The equilibrium chemical potential can be calculated using the Widom particle insertion method [15]. In spite of its simplicity of implementation (which has lead to its widespread use) it presents a serious drawback for the simulation of the adsorption of water and other fluids with highly directional potentials. This results from the volume exchange trials, which require the positions of all atoms to be rescaled. This is impractical for this type of system, since a change to the relative position of the water 
molecules would bring about a serious disruption of the hydrogen bonded network, leading to an almost certain rejection of the trial. Furthermore, in a situation where polar surface sites are fixed on the surface of the pore (which is the situation of fundamental and practical interest in the case of water adsorption), a volume exchange would cause an unphysical change in the relative position of these sites.

Molecular dynamics (MD) in the canonical ensemble (constant $N, V$ and $T$ ) has also been applied to study phase equilibrium. One approach [21] essentially involves the quenching of the fluid in the pore from a supercritical to a subcritical temperature. The fluid will then, given enough time, equilibrate into two distinct phases separated by an interface. An alternative method is to simply simulate a large pore at a density that is intermediate between liquid and vapour [22,23]. Provided that the system is large enough and is allowed sufficient equilibration time, it will also separate into two distinct phases. In both cases, one must then calculate the chemical potential using, for example, the particle insertion method. The advantages of this method are that it provides dynamic information about diffusion in the pore and kinetics of the phase transition, as well as showing details of the interface itself. The authors also suggest the possibility of using a canonical Monte Carlo (CMC) simulation instead of MD (although in this case one will not obtain any dynamic information). This approach, however simple in principle, requires large system sizes and very long equilibration periods, which limit its applicability to water simulations.

Some other variants of the MC method have also been developed for the simulation of phase equilibrium. One of these is the isotension ensemble MC [24], which also involves volume changes and is thus impractical for the same reasons as the GEMC. A different type of approach is the basis of the histogram reweighting method $[25,26]$. Essentially, this involves calculating the total energy and density over 
a series of GCMC simulations and then collecting them in the form of histograms. These histograms allow for the construction of the grand canonical partition function, from which it is possible to calculate the equilibrium phase transition pressure [27]. This method is particularly useful in the vicinity of the critical point, where density fluctuations are large (thus allowing for the construction of the histograms with only a few runs), but becomes much more computationally demanding at lower reduced temperature, where a large number of simulations is required to accurately construct the histograms.

An alternative way of calculating the equilibrium phase transition by thermodynamic integration was proposed recently by Neimark and Vishnyakov [18]. Their so-called gauge-cell method is, at its core, similar to the pore-fluid Gibbs ensemble method [20], but the volume of the bulk (or "gauge") cell and the total number of molecules are adjusted so as to restrict the density fluctuations inside the pore. This enables one to plot the entire van der Waals loop, including the metastable vapour and liquid branches as well as the unstable branch of the isotherm. We thus obtain a reversible path between the vapour and liquid phases that can be integrated to obtain the point of equal free energy. It is possible to show [18] that this condition is satisfied by Maxwell's construction of equal areas. Further support to the validity of this type of approach is given by the work of Corti and Debenedetti [28], who performed Monte Carlo simulations of bulk vapour-liquid equilibrium using a "restricted ensemble". They also obtained continuous van der Waals loops at subcritical conditions and went on to show that the results are independent of the severity of the constraint (provided it is severe enough to prevent incipient phase separations). These authors concluded that the van der Waals loops thus obtained are 
the true equation of state of a constrained system and not simply an artefact of the simulation method [28].

This type of GEMC simulation does not require mechanical equilibrium between the pore and gauge cells due to the tensorial nature of the pressure in the pore [20], so there is no need for the inconvenient volume exchange steps. The gauge-cell method was compared to both the Peterson and Gubbins method of integration and the pore-pore GEMC method by Neimark and Vishnyakov [18] and was found to yield consistent results in all cases examined. Furthermore, it required significantly less computational effort than the Peterson and Gubbins method of integration, while also providing information about the metastable and unstable branches of the isotherm (such as limits of stability of the vapour and liquid phases). Although it does possess some drawbacks (see the next section for details), it seems to be the most suitable method for our purposes.

\section{Simulation Details}

Water is represented by the SPC/E potential [29]. This model is composed of a Lennard-Jones site and a negative charge at a location corresponding to the oxygen atom in the water molecule, and two positive point charges located at the positions of the hydrogen atoms. Geometric and potential parameters were taken from Berendsen et al. [29]. Methane molecules are represented by a single Lennard-Jones site, with parameters taken from Hirschfelder et al. [30]. Each pore wall is represented by an infinite number of graphitic layers composed of Lennard-Jones sites. The pore width (w) is defined as the distance between the centres of the carbon atoms of the innermost layers. Furthermore, it is assumed that the walls are structureless and the carbon atoms are smeared out uniformly over the graphitic layer. The potential between an adsorbate molecule and this smooth carbon surface is thus given by the 
10-4-3 potential of Steele [31]. Solid-fluid Lennard-Jones parameters were calculated using the Lorentz-Berthelot combining rules. Potential parameters for water, methane and graphite are summarised in table 1. [Insert table 1 about here]

We have used GCMC simulations to calculate adsorption and desorption isotherms in pores of several widths. The simulation cell, representing the slit pore, is rectangular, bounded in the $z$ direction by the pore walls and replicated up to infinity in the $x$ and $y$ directions by employing periodic boundary conditions. Unless otherwise stated, the number of equilibration steps for each point of the adsorption and desorption isotherms was $1 \times 10^{7}$. The sampling period was divided into 20 blocks of at least $10^{5}$ steps to obtain a set of independent samples, from which an overall average density and a standard deviation were calculated. Each type of MC trial (displacement, creation and destruction) was chosen randomly with the same probability. In the case of water (a multi-site molecule) the displacement trial entailed a random rotation as well as a translation of the molecule. Since the potential between charged molecules is long-ranged in nature, special techniques must be employed during water simulations to account for interactions beyond the central periodic cell. We have found the method of Heyes and van Swol [32] to be the most efficient for handling long-range interactions in this type of application. Further details concerning the efficient implementation of the GCMC algorithm for water adsorption studies were given in a previous publication [33].

The gauge-cell method requires two separate simulation cells at the same constant temperature, one representing the pore and another representing the bulk fluid reservoir of limited capacity. The pore cell was identical to the one used in the GCMC method and is described above. The gauge cell was cubic in geometry and was replicated up to infinity in all three directions by employing periodic boundary 
conditions. Each type of trial (displacement in each cell and particle exchange between both cells) was randomly chosen with equal probability. The acceptance criteria for both trials are identical to those of the pore-fluid GEMC [20], so they shall not be repeated here. For each point of the isotherm, the system was allowed to equilibrate for at least $6 \times 10^{6}$ steps after which the densities in both cells were calculated using an analogous procedure to the GCMC simulations, with a minimum sampling block size of $4 \times 10^{5}$ steps. Long-range interactions in the water simulations were accounted for using the method of Heyes and van Swol [32] for the pore cell and using the standard three-dimensional Ewald sums [1] for the gauge cell.

The total system, comprising both simulation cells, is, strictly speaking, in the canonical ensemble (i.e., the total $N, V$ and $T$ are constant). Thus, at equilibrium, the total Helmholtz free energy $(F)$ is at a minimum. The fact that the values of $F$ and $N$ for the total system are the sum of their individual components in each cell (from here on, quantities referring to the gauge cell will be denoted by a subscript "g" while quantities relating to the pore will have a subscript "p") leads to the equilibrium condition:

$$
\mu=\left.\left(\frac{\partial F_{g}}{\partial N_{g}}\right)\right|_{V_{g}, T}=\left.\left(\frac{\partial F_{p}}{\partial N_{p}}\right)\right|_{V_{p}, T}
$$

The stability criterion for the total system is

$$
\left.\left(\frac{\partial^{2} F_{p}}{\partial N_{p}^{2}}\right)\right|_{V_{p}, T}+\left.\left(\frac{\partial^{2} F_{g}}{\partial N_{g}^{2}}\right)\right|_{V_{g}, T}>0
$$

The fulfilment of this condition, however, does not require that the fluid inside the pore be stable [18]. If one wishes to sample the unstable branch of the isotherm, equation (2) implies (assuming that the fluid in the gauge cell is itself stable) that 


$$
\left.\left(\frac{\partial^{2} F_{g}}{\partial N_{g}^{2}}\right)\right|_{V_{g}, T}>-\left.\left(\frac{\partial^{2} F_{p}}{\partial N_{p}^{2}}\right)\right|_{V_{p}, T}
$$

Substituting equation (1) for the chemical potential, we obtain, after manipulation,

$$
\frac{V_{g}}{V_{p}}<\left.\left(\frac{\partial \rho_{p}}{\partial \mu}\right)\right|_{T} /\left.\left(\frac{\partial \rho_{g}}{\partial \mu}\right)\right|_{T}
$$

where $\rho$ is the density. Equation (4), originally presented by Neimark and Vishnyakov [18], imposes restrictions on the size of the gauge cell in order to allow for the sampling of unstable points.

This criterion is written in terms of the chemical potential. However, in most practical situations, the relevant thermodynamic variable is the pressure in the bulk phase $(P)$. In the original pore-fluid Gibbs ensemble method [20], the chemical potential was calculated during the simulation by the particle insertion method, and this could in turn be related to the pressure. In the gauge-cell method, as proposed by Neimark and Vishnyakov [18], the pressure was instead calculated from the density in the gauge cell. This can be accomplished by using an appropriate equation of state or by performing a series of independent simulations in the gauge cell alone. By performing such a set of simulations, we have observed that the fluid in the gauge cell behaves essentially as an ideal gas for all the conditions of relevance to this study. Thus, the pressure can be calculated from the density in the gauge cell using the ideal gas law,

$$
P=\rho_{g} k_{B} T
$$

where $k_{B}$ is Boltzmann's constant. For each individual point on the isotherm, the final number of molecules in each cell are related by

$$
N=N_{p}+N_{g}
$$


since the total number of molecules in the system must remain constant throughout the simulation. It follows from equation (6) that:

$$
\rho_{p}=\frac{N}{V_{p}}-\frac{V_{g}}{V_{p}} \rho_{g}
$$

Substituting equation (5) into the above expression we arrive at:

$$
\rho_{p}=\frac{N}{V_{p}}-\frac{V_{g}}{V_{p} k_{B} T} P
$$

From this expression, we can see that all the possible outcomes of each run lie on a straight line in a pressure vs. density plot, since all other variables remain constant. This is represented schematically by the dashed line in figure 1. Depending on the fixed value of $N$ and $V_{g}$, this trajectory of possible outcomes will intersect the isotherm in one or more points. Those variables should be adjusted so that there is only one point of intersection, but this is difficult to achieve in practice since we do not know what the isotherm looks like a priori. If there are multiple points of intersection, the simulation will converge to the most stable configuration and this makes it difficult to sample the unstable branch of the isotherm. The precise criterion for sampling in the unstable region is given by equation (4). Graphically, this means that the slope of the trajectory of possible outcomes must be smaller than the local derivative of the unstable branch of the isotherm. The trajectory represented in figure 1 shows a case where this criterion is satisfied. The final result of this run will lie at point $\mathbf{J}$. In practice, the tuning of $N$ and $V_{g}$ is a process of trial and error. A few initial runs will provide a rough sketch of the van der Waals loop, and this can be refined by more simulations for which it will be easier to determine the appropriate values of $N$ and $V_{g}$. It is interesting to note that equation (8) reduces to a constant pore density, corresponding to the canonical ensemble, when $V_{g}=0$ (a horizontal line in the diagram of figure 1). In fact, it is possible to obtain the van der Waals loop by a 
canonical MC simulation, but in this case the chemical potential must be determined using the particle insertion method, which suffers from accuracy problems [18].

After obtaining the entire isotherm, it is simply a case of performing a numerical integration to obtain the point of equilibrium phase coexistence. This can be calculated by Maxwell's rule of equal areas [18]:

$$
\int_{\mu_{e q}}^{\mu_{v s}} \rho_{a} d \mu-\int_{\mu_{l s}}^{\mu_{v s}} \rho_{u} d \mu+\int_{\mu_{e q}}^{\mu_{l s}} \rho_{d} d \mu=0
$$

In the above equation, subscript $a$ refers to the adsorption branch, subscript $u$ refers to the unstable branch, subscript $d$ refers to the desorption branch, subscript $v s$ refers to the vapour spinodal point, subscript $l s$ refers to the liquid spinodal point and subscript $e q$ refers to the point of equilibrium phase coexistence. For the integration procedure, we have simply used the trapezoidal rule, since the possible errors will be smaller that the errors inherent to the simulation method.

We have also performed some Monte Carlo simulations in the canonical ensemble, mainly as a check of the results of the gauge-cell method. In a CMC simulation, $N, V$ and $T$ are kept constant and the system equilibrates by virtue of displacement trials alone. In this type of simulation, the conjugate variables $\mu$ and $N$ switch the roles they have in a GCMC simulation; here the density is fixed and the chemical potential is calculated during the run. To calculate the chemical potential, we have used the particle insertion method due to Widom [34]. This essentially involves the random creation of a test particle uniformly over the pore volume at frequent intervals during the simulation. The expression for the calculation of the chemical potential in a slit-shaped pore is [35]:

$$
\mu=3 \ln \Lambda+\ln \langle\rho(z)\rangle-\ln \left\langle\exp \left[-\frac{U(z)}{k_{B} T}\right]\right\rangle
$$


In the above expression, $\Lambda$ is the thermal de Broglie wavelength, $U$ is the potential arising from the interaction of the test particle with all the other molecules in the system, and the angular brackets denote an ensemble average. When the system reaches equilibrium the last two terms in equation (10), although strongly dependent on $z$, should yield a constant when added. One must note, however, that this method runs into accuracy problems when the density is close to zero (such as near the centre of the pore when adsorption is confined to a monolayer on each pore wall) or very high (as in a dense adsorbed layer). At very low density there is a large uncertainty in the calculation of the second term on the right-hand side of equation (10), whereas if the density is too high, the insertion of the test particle becomes extremely difficult and the average of the last term on the right-hand side of equation (10) is inaccurate. These aspects will be discussed in more detail in the next section.

\section{Results and Discussion}

In figure 2 we show adsorption isotherms for methane in a slit pore of width 2 $\mathrm{nm}$ at a temperature of $120 \mathrm{~K}$. This corresponds to a reduced pore width $\left(w_{r}=w / \sigma_{f}\right.$, where $\sigma_{f}$ is the Lennard-Jones molecular diameter for the adsorbate) of 5.2, and a reduced temperature $\left(T_{r}=T / T_{c}\right.$, where $T_{c}$ is the critical temperature $)$ of 0.63 . The pressure is scaled by the bulk saturation pressure $\left(P_{\text {sat }}\right)$. These isotherms were obtained from GCMC simulations with different lengths of equilibration period. In all isotherms, we can identify four distinct regions. At very low pressure, there is a steep rise in adsorption (closed symbols), corresponding to the formation of a monolayer on the surface of the carbon. After this monolayer is complete, there is little further adsorption until the pressure of spontaneous condensation $\left(P_{s c}\right)$ is reached. At this point, the fluid abruptly jumps from a vapour-like to a liquid-like state, remaining in 
this state as the pressure is further increased. If we start from a configuration in the liquid state and gradually reduce the pressure, we can obtain a desorption isotherm (open symbols). As the pressure is lowered, the system remains in the liquid state down to the point of spontaneous evaporation $\left(P_{s e}\right)$. As expected, the GCMC isotherms show hysteresis in the region of phase equilibrium. However, the results in the vicinity of the phase transition are highly dependent on the length of the equilibration period. As we can see from figure 2, the size of the hysteresis loop decreases with the number of equilibration steps until it almost vanishes for very long simulations. This supports the hypothesis that with an infinite-sized GCMC run the true equilibrium transition would in principle be attainable. These observations are in accordance with the findings of Schoen et al. [16]. We have also checked for finitesize effects by performing GCMC runs with different sizes of the simulation cell. We have observed the results to be invariant (within statistical errors) when the lateral dimensions of the cell are larger that the width of the pore. If the lateral dimensions are equal to or smaller that the pore width, the results are no longer reliable; the system was observed to oscillate between the two phases for a wide range of pressures and this did not allow for adequate sampling. A similar effect was also observed by Schoen et al. [16] in their MC studies. In all subsequent GCMC simulations, we have used a lateral dimension of $3 \mathrm{~nm}$ for the simulation cell. [Insert figure 2 about here]

We now turn our attention to the results obtained from a series of simulations using the gauge-cell method for the same system (figure 3 shows a close-up of the isotherm in the region of phase equilibrium). [Insert figure 3 about here] With this method, we were able to trace the entire van der Waals loop, including stable, metastable and unstable points. This enabled us to calculate the equilibrium transition pressure $\left(P_{e q}\right)$ by thermodynamic integration (as described in the previous section). 
The value obtained lies between $P_{s c}$ and $P_{s e}$, which indicates that both the vapour and the liquid phases remain metastable during GCMC adsorption and desorption runs, respectively. The results of the gauge-cell method overlap nicely with the GCMC simulations in the stable and metastable regions, which confirms the consistency of the method. They are also, as expected, independent of the size of the gauge cell (see figure 3). From an analysis of the error bars, we have concluded that in order to reduce the statistical errors, $V_{g}$ should be as large as possible (while still avoiding multiple intersection points with the isotherm). We have also investigated the effect of the lateral dimensions of the pore cell on the gauge-cell results. figure 4 shows results obtained from simulations using three different values of $V_{p}$. The results are statistically indistinguishable for the two largest cells, but, as in the case of the GCMC results, we find a marked difference when the cell is cubic. [Insert figure 4 about here] We have therefore used a lateral dimension of $3 \mathrm{~nm}$ for the pore cell in all subsequent simulations. To further check the results of the gauge-cell method, we have traced the van der Waals loop using CMC simulations together with the particle insertion method. These results are shown in figure 5. The loops overlap within statistical error, which once more confirms the validity of the gauge-cell calculations. [Insert figure 5 about here] In the CMC method, it was difficult to obtain accurate results, due to the problems inherent to the particle insertion method at both very low and very high densities. By plotting chemical potential profiles obtained with this method for different sampling sizes (figure 6) it is possible to see that only for very long CMC runs is $\mu$ essentially constant across the whole pore. [Insert figure 6 about here] We have also checked the value of $P_{e q}$ obtained with the gauge-cell method by comparing it to the outcome of a CMC simulation in a very large pore (lateral dimensions of 7.5 $\mathrm{nm})$. This simulation follows the prescription of Heffelfinger and van Swol [21,22] 
for calculating the chemical potential at the equilibrium phase transition, as described in section 3. In the previous CMC simulations with a small periodic cell, the system was forced to remain homogeneous and we were thus able to plot the van der Waals loop. If the cell is large enough, however, the system will tend to separate into two distinct phases [23]. The chemical potential of such a system, if calculated correctly, should yield the value of $P_{\text {eq }}$. In figure 7 we show a snapshot of a typical configuration seen during the CMC run. We can easily confirm that the system has indeed split into two distinct phases (vapour in the centre of the simulation cell and liquid towards the edges) separated by an interface. [Insert figure 7 about here]

In table 2 we present the values of $P_{e q}$ calculated by several simulation methods: integration of the van der Waals loop obtained by the gauge-cell method, integration of the loop obtained by CMC simulations imposing fluid homogeneity, and the outcome of a CMC simulation with phase separation. Also shown are the estimated errors and the approximate length of computer time required for each calculation. There is very good agreement between the values of $P_{e q}$ calculated by all three methods, but the gauge-cell method is by far the fastest. In light of this, the gauge-cell method is the most practical for the calculation of coexistence. In spite of the fact that it requires a certain amount of trial and error to sample the unstable region, it is still more accurate than the CMC method combined with integration of the van der Waals loop, which suffers from the already mentioned difficulties associated with the particle insertion method. The CMC simulation with phase separation provides a straightforward, easy to implement method. However, in such a large and heterogeneous system, constancy of the chemical potential along the pore is difficult to achieve. In fact this particular run required a sampling size of $2 \times 10^{8}$ Monte Carlo steps. Such a long simulation becomes impracticable when dealing with polar 
molecules such as water, when computationally intensive long-range interactions must be evaluated. It is worth mentioning in passing that GCMC simulations for this system yielded (non-equilibrium) condensation and vaporisation pressures of $\mathrm{P} / \mathrm{P}_{\text {sat }}=$ 0.0750 and 0.0743 , respectively. For this combination of pore size and temperature, in which the hysteresis loop obtained with the GCMC method is very narrow, these values give a tight bound on the equilibrium transition pressure. [Insert table 2 about here]

A similar study of the vapour-liquid transition was performed for the same pore size at a temperature of $86 \mathrm{~K}\left(T_{r}=0.45\right)$, and the results are presented in figure 8. As we are now further away from the critical temperature, the extent of metastability is larger, as well as the hysteresis loop. In fact, the GCMC desorption branch now extends virtually down to the liquid spinodal point. This effect is due to the fact that at a lower temperature the fluctuations are reduced, and therefore the system remains trapped in metastable configurations for longer. In this case, longer equilibration periods resulted in an almost insignificant reduction of the size of the hysteresis loop. It should be noted that at this temperature methane is likely to exhibit freezing transitions [36]. However, we did not analyse this phenomenon in detail, since the main purpose of our methane simulations is a direct comparison with the behaviour of water. [Insert figure 8 about here]

In figure 9 we present results using both GCMC and the gauge-cell method for water adsorbed in a $1.64 \mathrm{~nm}$ graphitic pore at $293 \mathrm{~K}$. This represents equivalent reduced conditions $\left(w_{r}\right.$ and $\left.T_{r}\right)$ to those of the methane simulation at $86 \mathrm{~K}$, in order to allow for direct comparison between the results. It becomes clear at once that the qualitative behaviour of water adsorbed in a graphitic pore is significantly different from that of methane. The first obvious difference in the GCMC results is that there is 
hardly any water adsorbed in the pore at low pressure. The graphite pore is hydrophobic, which is due to a very low fluid-solid affinity in this system. Furthermore, the spontaneous condensation occurs long before any monolayer is formed, and the transition is from an essentially empty pore to a pore filled with liquid water. In fact, the spontaneous condensation pressure is even larger than the bulk saturation pressure at $293 \mathrm{~K}$, which is also a consequence of the hydrophobicity of the graphite pore. Once more, we observe pronounced hysteresis, with the desorption curve extending almost down to the limit of stability of the liquid phase. This behaviour of water adsorbed in carbon pores qualitatively agrees with experimental results [1], as well as with independent molecular simulation studies [8,9] using different water models. [Insert figure 9 about here]

We have obtained the corresponding van der Waals loop from a series of simulations with the gauge-cell method and proceeded to calculate $P_{e q}$ for water. A check of finite size effects for the water isotherm has also been performed (results not shown here) and the same conclusions as in the case of methane have been reached. It is worth noting that the error bars in the lower density part of the loop, close to the vapour spinodal, are quite large. This originates from the need to use a very small gauge cell in order to obtain points in this part of the unstable branch (due to the very small local gradient of the unstable branch is this range - see section 3). Indeed, the unusual shape of the loop makes sampling in this region rather difficult, so only with relatively long simulation runs is one able to obtain accurate results in the vicinity of the vapour spinodal.

From the results we can see that the limit of stability of the vapour phase extends to very high pressures, substantially above the bulk saturation pressure. However, the equilibrium transition pressure lies well below $P_{\text {sat. }}$ Thus, the effect of 
confinement is to reduce the transition pressure relative to that of the bulk. In the case of water confined between hard walls (i.e. with no attractive part of the potential), the opposite effect has been observed by simulation [12]. However, the attractive potential of the carbon walls, although weak compared to the water-water interaction, seems to be strong enough to bring about a shift of the vapour-liquid transition to lower pressures.

The differences between the behaviour of water and methane can be explained based on the different mechanisms for condensation. To aid visualisation of this phenomenon, we have calculated density profiles across the pore for various values of the average density (marked by arrows in figure 8 and figure 9). These are presented in figure 11 for methane and figure 13 for water, while corresponding snapshots of typical configurations obtained during each run are shown in figure 10 for methane and figure 12 for water. As we have seen, adsorption of methane at low pressure occurs only at the pore walls (figure 10a) until a complete monolayer is formed (figure 10b). As the pressure is increased further, the adsorbate tends to form a second layer parallel to the existing layer (figure 10c). However, this configuration is already unstable. In fact, as soon as the density fluctuations are large enough to cause the layers on opposite sides of the pore to connect, spontaneous condensation occurs. The stable phase is a liquid-filled pore (figure 10d). [Insert figure 10 about here] This mechanism of condensation, which occurs near the end of the metastable vapour branch, is similar to that observed by Heffelfinger et al. [22] in their MD simulations of cylindrical pores and by Müller and Gubbins [10] in slit-shaped carbon pores, and agrees with the approach of Saam and Cole [37] based on instability and metastability of adsorbed films. The density profiles (figure 11) start building up near the walls at first and only when the monolayer is complete is there any significant adsorption near 
the centre of the pore. That is why in a GCMC simulation the second layer is not formed before the pore fills with liquid (in a larger pore, a second adsorbed layer would be able to form before the onset of condensation). [Insert figure 11 about here]

The behaviour described above is typical of a system in which there is a large affinity between the solid and the adsorbate. In the case of water on graphite, the opposite is observed. Due to the possibility of formation of hydrogen bonds with each other, water molecules tend to cluster together. The formation of an adsorbed monolayer is therefore energetically unfavourable. The pore is initially empty until a small cluster of water molecules appears (figure 12a). This allows more water molecules to bond to the cluster, until it spans the whole pore width (figure 12b). It then extends laterally across the pore (figure 12c) and finally the liquid phase is reached (figure 12d). [Insert figure 12 about here] All the density profiles (figure 13) have basically the same shape along the $z$ direction, but they simply extend laterally as the adsorbed density in the pore increases. One might say that the methane adsorbed phase "grows" from the walls inward, while the water "grows" laterally along the pore. This has an effect on the structure of the liquid phase, which is different for water and methane (one can see this by comparing the density profiles at the highest average density for the two species in figure 11 and figure 13), once more in agreement with previous simulation results [8]. It should be noted, however, that all the water configurations illustrated (except, of course, the empty and the liquid-filled pore) are unstable and are never observed in a GCMC isotherm. Instead, the pore remains empty until density fluctuations are large enough to allow the formation of a pore-spanning cluster, after which the pore instantaneously fills with liquid. This also explains the large extent of metastability of the vapour phase in water adsorption, since the variation in density that must be achieved to jump from the vapour to the 
liquid phase is extremely large, and this only becomes possible at very high pressures. In the case of methane, on the other hand, there is only a small distance to be covered after the monolayer is formed, and therefore the onset of liquid formation occurs at a much lower relative pressure. [Insert figure 13 about here]

To complete our analysis, we have examined the effect of pore width on the phase equilibrium of water. The isotherms obtained using the gauge-cell method for three different pore sizes are shown in figure 14. It can be seen that a reduction of $w$ brings about a reduction in $P_{e q}$, a decrease in the extent of vapour metastability and also a decrease in the pressure of spontaneous condensation of the GCMC isotherm. An increase in the pore width means that it is harder for a pore-spanning cluster to form. The system remains trapped in the vapour phase for longer, until high pressure allows for density fluctuations of enough magnitude to overcome the large energy barrier between the two phases. In a smaller pore the attractive parts of the solid-fluid potential for the individual walls overlap, and this effect, associated to the reduction of the physical distance between the walls, leads to an earlier onset of condensation. The increase of $P_{e q}$ with $w$ is caused mainly by a greater extent of vapour metastability, since the liquid spinodal is seen to be almost independent of the pore width. This might seem surprising, but in fact, once the liquid phase is formed, its stability is mainly dictated by liquid-liquid rather than liquid-solid interactions. Thus the distance between the pore walls exerts little influence on the limit of stability of the liquid phase. Effectively, this means that there will be a lower limit to the pressure at which a hysteresis loop in a real adsorbent will close. This limit will be dictated solely by thermodynamic considerations and will be essentially independent of the pore size. [Insert figure 14 about here] 


\section{Conclusions}

In this paper we have applied grand canonical, canonical and gauge-cell Monte Carlo simulations to study the vapour-liquid equilibrium of water and methane in graphitic pores. The gauge-cell method was shown to be a useful method for this type of study, since it provides information not only about the phase coexistence properties, but also about the limits of stability of the vapour and liquid phases. A detailed description of the implementation of the method was given and a check of the results against $\mathrm{CMC}$ simulations was also performed. The gauge-cell method possesses several advantages relative to more widely employed methods (such as the Peterson and Gubbins thermodynamic integration and the pore-pore Gibbs ensemble method), the most important for our case being the possibility of application to chemically heterogeneous pores using relatively expedient simulations. However, it still requires a certain amount of trial and error and close monitoring of the results of individual runs. Nevertheless, it seems to be the most promising method available to date for our purposes.

We have, for the first time, presented simulation results for water in carbon pores at the equilibrium phase transition and compared them with methane adsorption at the same reduced conditions. The behaviour of these two fluids was observed to be quite different in nature. The strong solid-fluid attraction in the case of methane induced the adsorption of a dense monolayer at the surface prior to condensation. At high enough pressure, density fluctuations inside the pore cause the two adsorbed layers to connect, forming a liquid bridge. After this, the pore instantaneously fills with liquid. In the case of water, the solid-fluid interactions are much weaker than the hydrogen bonds established between fluid molecules. This means that water molecules tend to cluster together inhibiting the formation of a monolayer. The 
formation of a large cluster that connects both pore walls seems to be critical in the process of condensation, the transition being from an essentially empty pore to a pore filled with liquid water. The effect of pore width on water adsorption was also analysed. We have observed that a decrease of the pore width shifts the equilibrium transition to lower pressures and reduces the extent of vapour metastability. This is a combined result of the decrease of the physical distance between the pore walls and the enhancement of the solid-fluid attractive potential in narrow pores. However, the effect on the liquid metastable state seems to be almost negligible. This stems from the fact that in a closely packed liquid configuration, the fluid-fluid interactions are predominant over the solid-fluid interactions. The instability of the liquid is brought about by the formation of a large enough bubble of vapour inside the pore, and this process is little influenced by the solid-fluid interactions.

\section{Acknowledgements}

M. Jorge would like to acknowledge financial support from Fundação para a Ciência e Tecnologia -Portugal.

\section{References}

1. Rouquerol F., Rouquerol J. and Sing K., 1999, Adsorption by Powders and Porous Solids (London, Academic Press).

2. Evans R., 1990, J. Phys.: Condens. Matter, 2, 8989.

3. Evans R., Marconi U. M. B. and Tarazona P., 1986, J. Chem. Phys., 84, 2376.

4. Seaton N. A., Walton J. P. R. B. and Quirke N., 1989, Carbon, 27, 853.

5. Gelb L. D., Gubbins K. E., Radhakrishnan R. and Sliwinska-Bartkowiak M., 1999, Rep. Prog. Phys., 62, 1573. 
6. Spohr E., Trokhymchuk A. and Henderson D., 1998, J. Electroanal. Chem., 450, 281.

7. Luzar A. and Leung K., 2000, J. Chem. Phys., 113, 5836.

8. Ulberg D. E. and Gubbins K. E., 1995, Molec. Phys., 84, 1139.

9. Müller E. A., Rull L. F., Vega L. F. and Gubbins K. E., 1996, J. Phys. Chem., 100,1189

10. Müller E. A. and Gubbins K. E., 1998, Carbon, 36, 1433.

11. McCallum C. L., Bandosz T. J., McGrother S. C., Müller E. A. and Gubbins K. E., 1999, Langmuir, 15, 533.

12. Brodskaya E. N., Zakharov V. V. and Laaksonen A., 2001, Langmuir, 17, 4443.

13. Brovchenko I., Geiger A. and Oleinikova A., 2001, Phys. Chem. Chem. Phys., 3, 1567.

14. Allen M. P. and Tildesley D. J., 1989, Computer Simulation of Liquids (Oxford: Clarendon Press).

15. Frenkel D. and Smit B., 1996, Understanding Molecular Simulation (London: Academic Press).

16. Schoen M, Rhykerd C. L., Cushman J. H. and Diestler D. J., 1989, Molec. Phys., $66,1171$.

17. Peterson B. K. and Gubbins K. E., 1987, Molec. Phys., 62, 215.

18. Neimark A. V. and Vishnyakov A., 2000, Phys. Rev. E, 62, 4611.

19. Panagiotopoulos A. Z., 1987, Molec. Phys., 61, 813.

20. Panagiotopoulos A. Z., 1987, Molec. Phys., 62, 701.

21. Heffelfinger G. S., van Swol F. and Gubbins K. E., 1987, Molec. Phys., 61, 1381.

22. Heffelfinger G. S., van Swol F. and Gubbins K. E., 1988, J. Chem. Phys., 89, 5202. 
23. Papadopoulou A., van Swol F. and Marconi U. M. B., 1992, J. Chem. Phys., 97, 6942.

24. Forsman J. and Woodward C. E., 1997, Molec. Phys., 90, 637.

25. Ferrenberg A. M. and Swendsen R. H., 1988, Phys. Rev. Lett., 61, 2635.

26. Wilding N. B., 1995, Phys. Rev. E, 52, 602.

27. Kiyohara K., Gubbins K. E. and Panagiotopoulos A. Z., 1997, J. Chem. Phys., $106,3338$.

28. Corti D. S. and Debenedetti P. G., 1994, Chem. Eng. Sci., 49, 2717.

29. Berendsen H. J. C., Grigera J. R. and Straatsma T. P., 1987, J. Phys. Chem., 91, 6269.

30. Hirschfelder J. O., Curtiss C. F. and Bird R. B., 1954, Molecular Theory of Gases and Liquids (New York: Wiley).

31. Steele W. A., 1974, The Interaction of Gases with Solid Surfaces (Oxford: Pergamon Press).

32. Heyes D. M. and van Swol F., 1981, J. Chem. Phys., 75, 5051.

33. Jorge M. and Seaton N. A., 2002, Molec. Phys., 100, 2017.

34. Widom B., 1963, J. Chem. Phys., 39, 2808.

35. Rowlinson J. S. and Widom B., 1982, Molecular Theory of Capillarity (Oxford: Clarendon Press).

36. Jiang S., Rhykerd C. L. and Gubbins, K. E., 1993, Molec. Phys., 79, 373.

37. Saam W. F. and Cole M. W., 1975, Phys. Rev. B, 11, 1086. 
table 1: Potential parameters for water, methane and graphite.

\begin{tabular}{ccccc}
\hline & Site & $\sigma / \mathrm{nm}$ & $\varepsilon /\left(\mathrm{J}^{\mathrm{mol}}{ }^{-1}\right)$ & $q / e$ \\
\hline Water & $\mathrm{H}$ & 0.0 & 0.0 & +0.4238 \\
& $\mathrm{O}$ & 0.3166 & 650.2 & -0.8476 \\
\hline Graphite & $\mathrm{C}$ & 0.340 & 232.8 & 0.0 \\
\hline Methane & $\mathrm{CH}_{4}$ & 0.381 & 1232.1 & 0.0 \\
\hline
\end{tabular}


table 2: Reduced vapour-liquid equilibrium transition pressure for methane adsorbed in a $2 \mathrm{~nm}$ pore at $120 \mathrm{~K}$ calculated by several methods. Also shown are the estimated error in the calculations and the approximate computation time on a single processor.

\begin{tabular}{cccc}
\hline Method & $\mathrm{P}_{\mathrm{eq}} / \mathrm{P}_{\mathrm{sat}}$ & Estimated error & Time (hrs) \\
\hline Gauge-cell MC & 0.07464 & 0.00156 & 5.80 \\
\hline CMC (homogeneous system)+ & 0.07429 & 0.00351 & 26.19 \\
integration & & & \\
\hline $\begin{array}{c}\text { CMC (heterogeneous system with } \\
\text { phase separation) }\end{array}$ & 0.07456 & 0.00249 & 51.65 \\
\hline
\end{tabular}




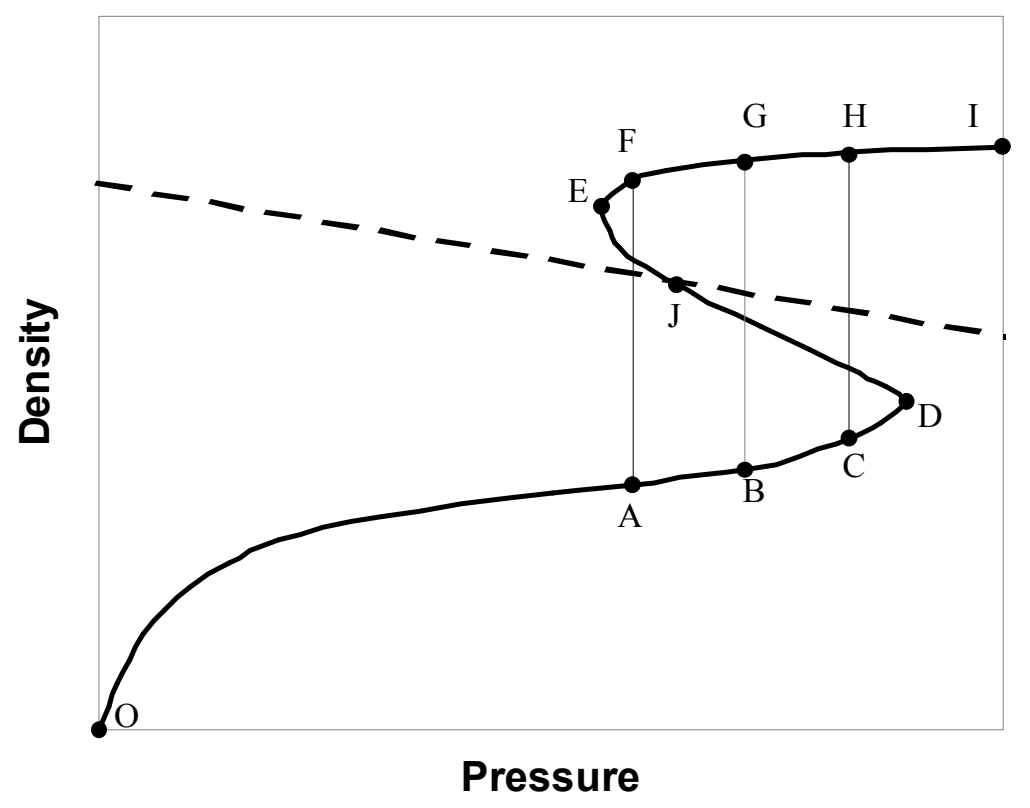

figure 1: Sketch of a typical isotherm at subcritical conditions. Segment $\mathrm{CH}$ represents the point of spontaneous condensation in a GCMC simulation; segment FA is the point of spontaneous evaporation in GCMC; segment BG shows the point of the true equilibrium transition; points $\mathrm{D}$ and $\mathrm{E}$ are the spinodal limits of stability of the vapour and liquid states, respectively; point I represents the end point of the adsorption run and the start of the desorption run; the dashed line shows a trajectory of possible outcomes of a gauge-cell simulation (for a given value of $\mathrm{N}$ and $\mathrm{Vg}$ ); and point $\mathrm{J}$ is where this trajectory intersects the unstable branch of the isotherm. 


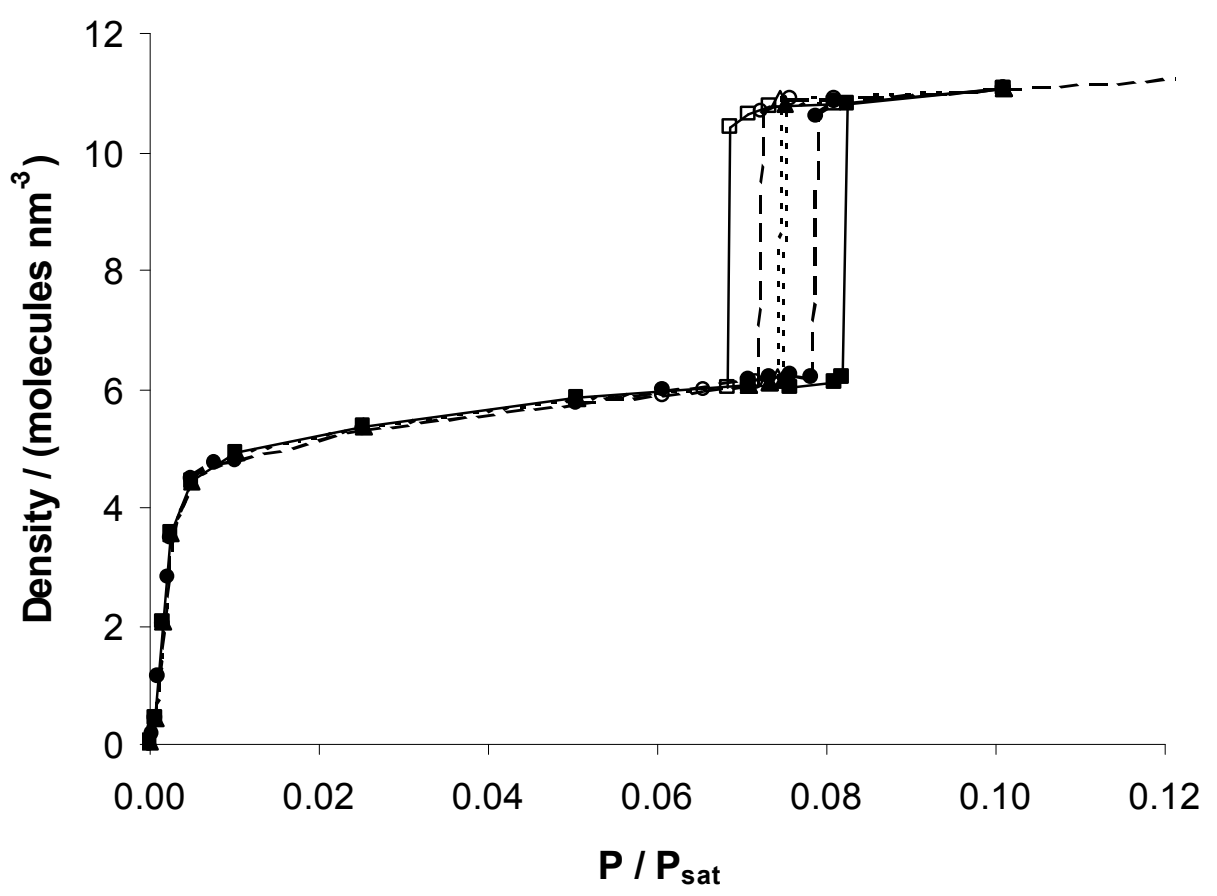

figure 2: GCMC isotherms for methane adsorbed in a slit pore of $2 \mathrm{~nm}$ in width, at a temperature of $120 \mathrm{~K}$. Squares (connected by a continuous line) were obtained from a simulation with $5 \square 105$ equilibration steps; circles (connected by a dashed line) correspond to an equilibration period of $2 \square 106$ steps; and triangles (connected by a dotted line) are for $1 \square 107$ equilibration steps. Filled symbols represent adsorption and open symbols represent desorption. 


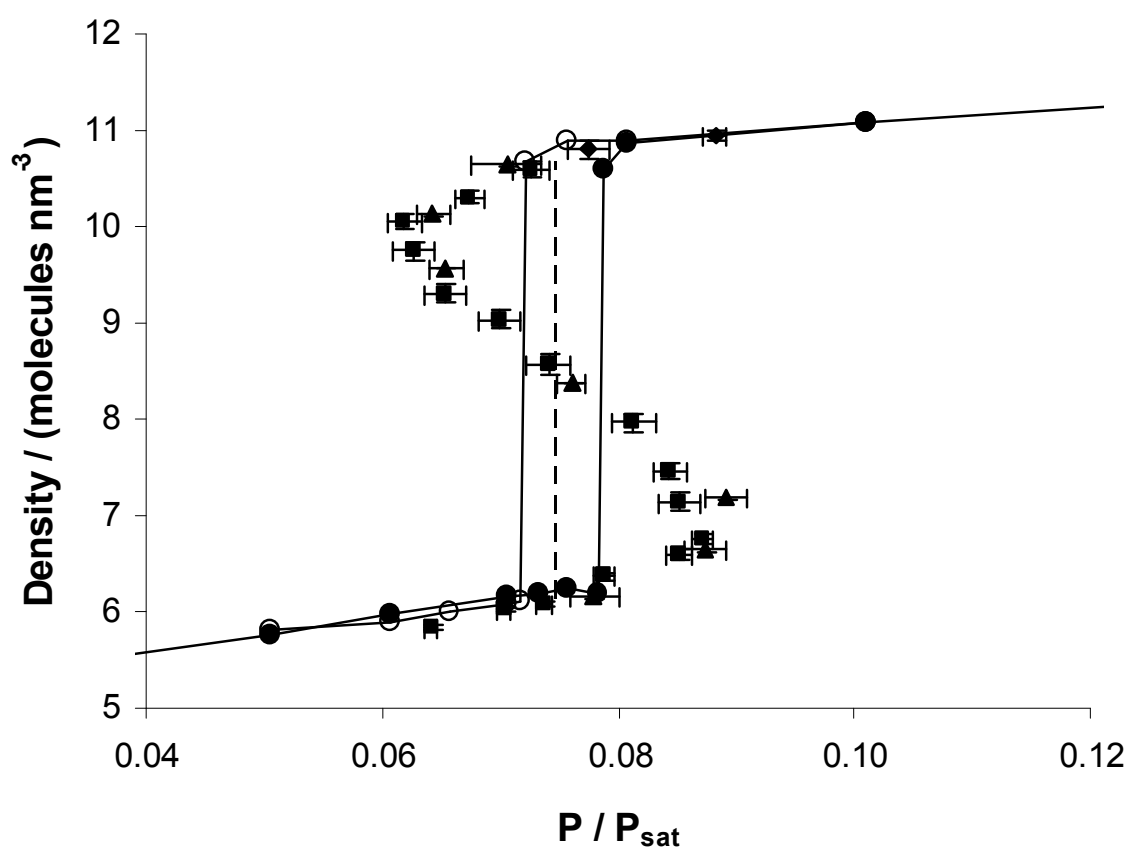

figure 3: Isotherm for methane adsorbed in a slit pore of $2 \mathrm{~nm}$ in width, at a temperature of $120 \mathrm{~K}$. Circles represent GCMC results with an equilibration period of $2 \square 106$ steps. This length was chosen to more clearly show the metastability of the vapour and liquid phases. Remaining symbols represent results of the gauge-cell method using different values of $\mathrm{Vg}$. The dashed line shows the true equilibrium phase transition. Error bars not shown are the size of the symbols used. 


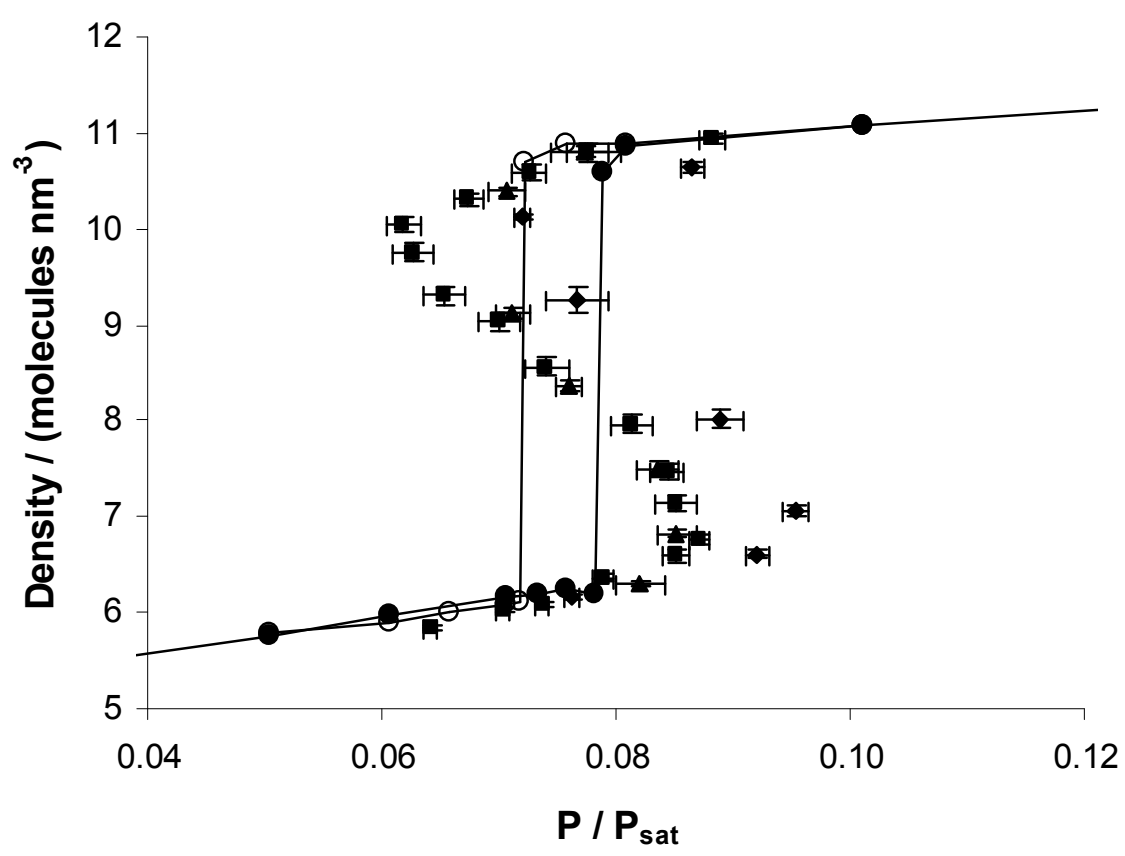

figure 4: Analysis of finite-size effects for methane in a $2 \mathrm{~nm}$ pore at $120 \mathrm{~K}$. Circles are the same as in figure 3 and remaining symbols are the results from the gauge-cell method at different lateral dimensions of the pore cell (Lp), as follows: diamonds, Lp $=2 \mathrm{~nm} ;$ squares, $\mathrm{Lp}=3 \mathrm{~nm} ;$ triangles, $\mathrm{Lp}=4 \mathrm{~nm}$. 


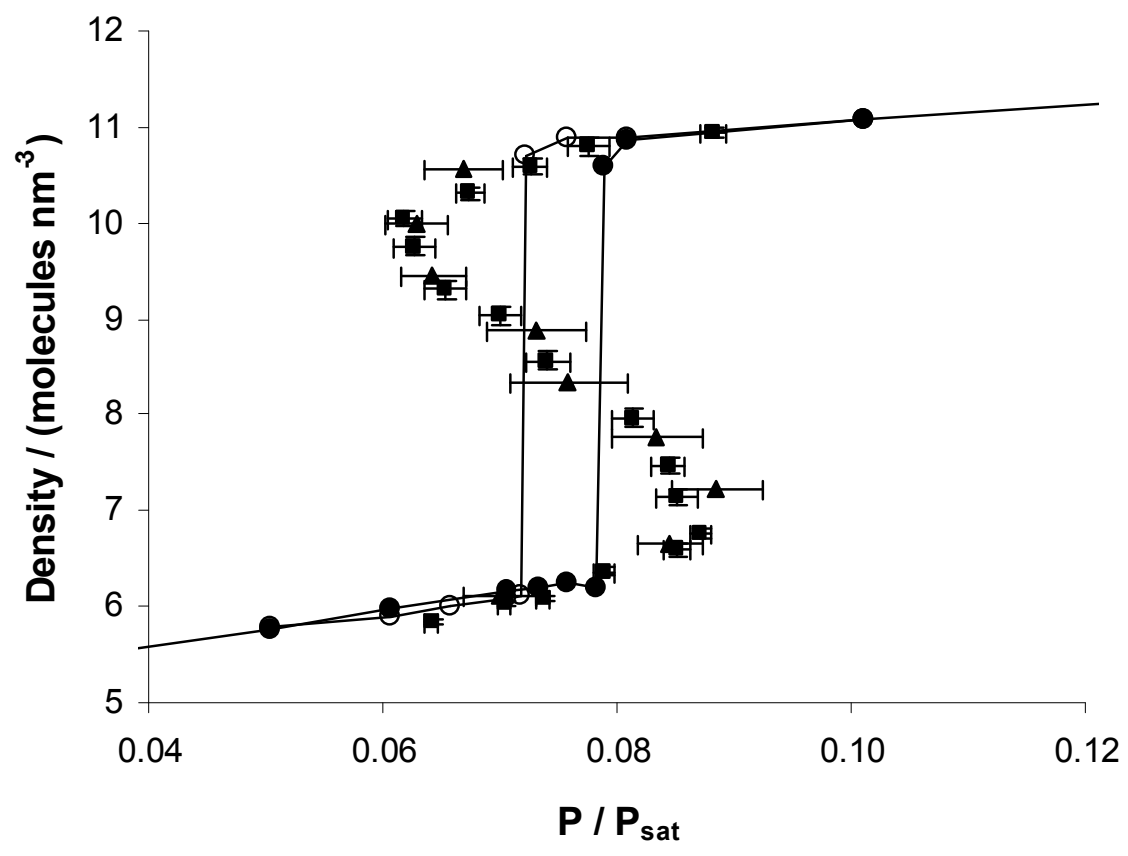

figure 5: Comparison between the gauge-cell method and CMC for methane at $120 \mathrm{~K}$ in a $2 \mathrm{~nm}$ pore. Circles are the same as in figure 3, squares represent the results of the gauge-cell method and triangles represent the loop obtained from CMC simulations with a small simulation cell. 


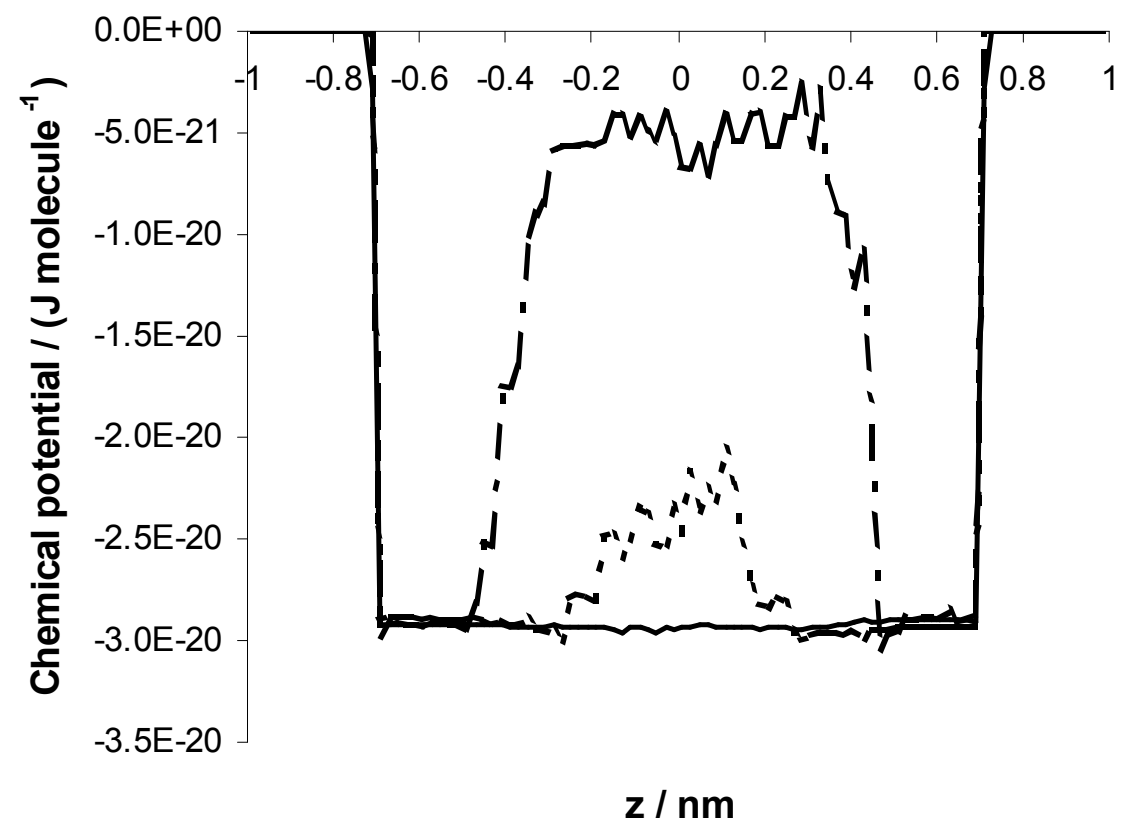

figure 6: Chemical potential profiles for methane at $120 \mathrm{~K}$ across a $2 \mathrm{~nm}$ pore, obtained from CMC simulations. The dashed-dotted line shows the results for a sampling period of $1 \square 106$ Monte Carlo steps, the dotted line is for $1 \square 107$ steps and the continuous line corresponds to $1 \square 108 \quad$ steps. 


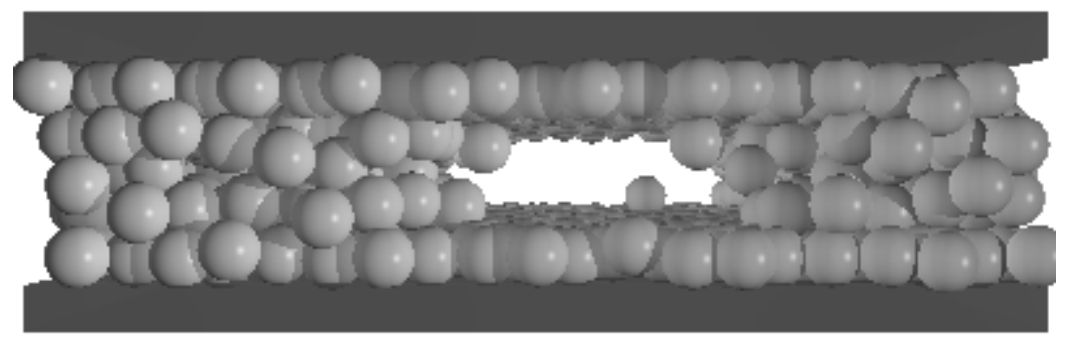

figure 7: Snapshot of a typical configuration obtained during the CMC simulation with a large periodic cell. Methane molecules are represented by the light grey spheres, while the graphite pore walls are shown in dark grey. 


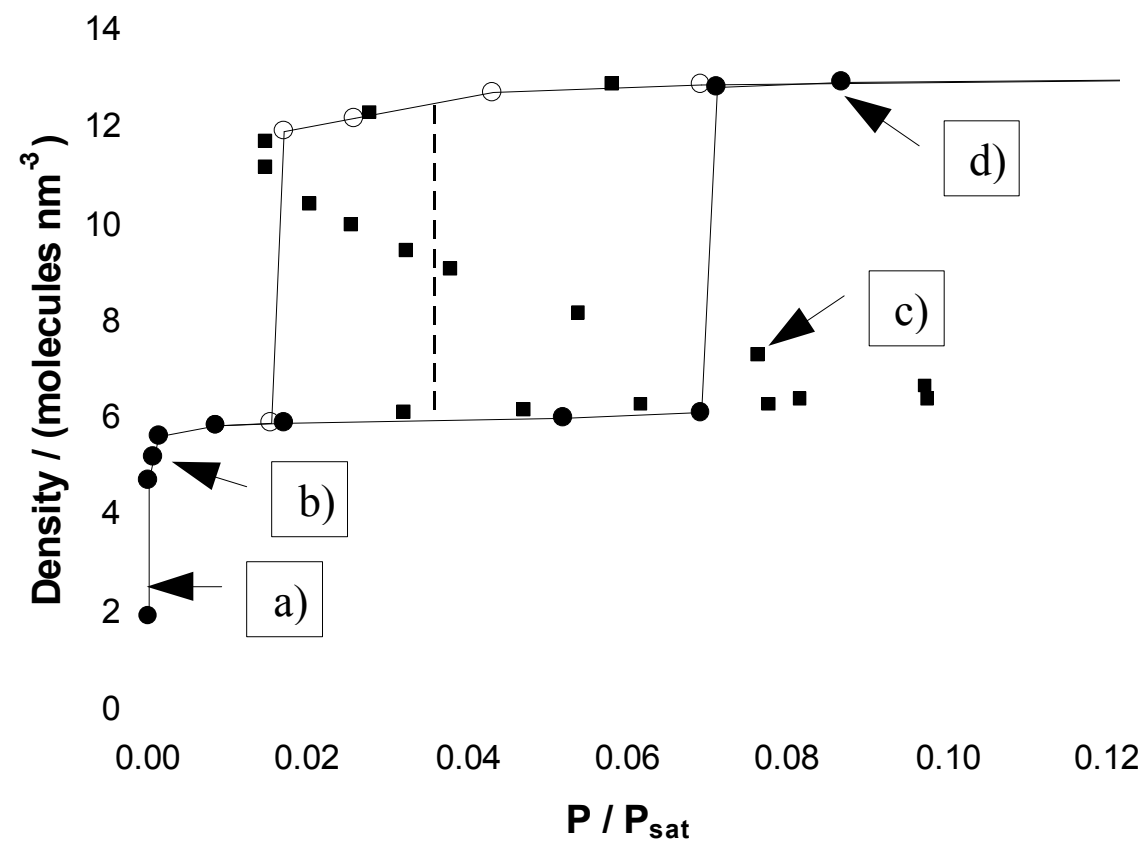

figure 8: Isotherm for methane adsorbed in a $2 \mathrm{~nm}$ pore at $86 \mathrm{~K}$. Symbols are the same as in figure 3. The small arrows and the labels mark the points at which density profiles and snapshot configurations were obtained. 
30

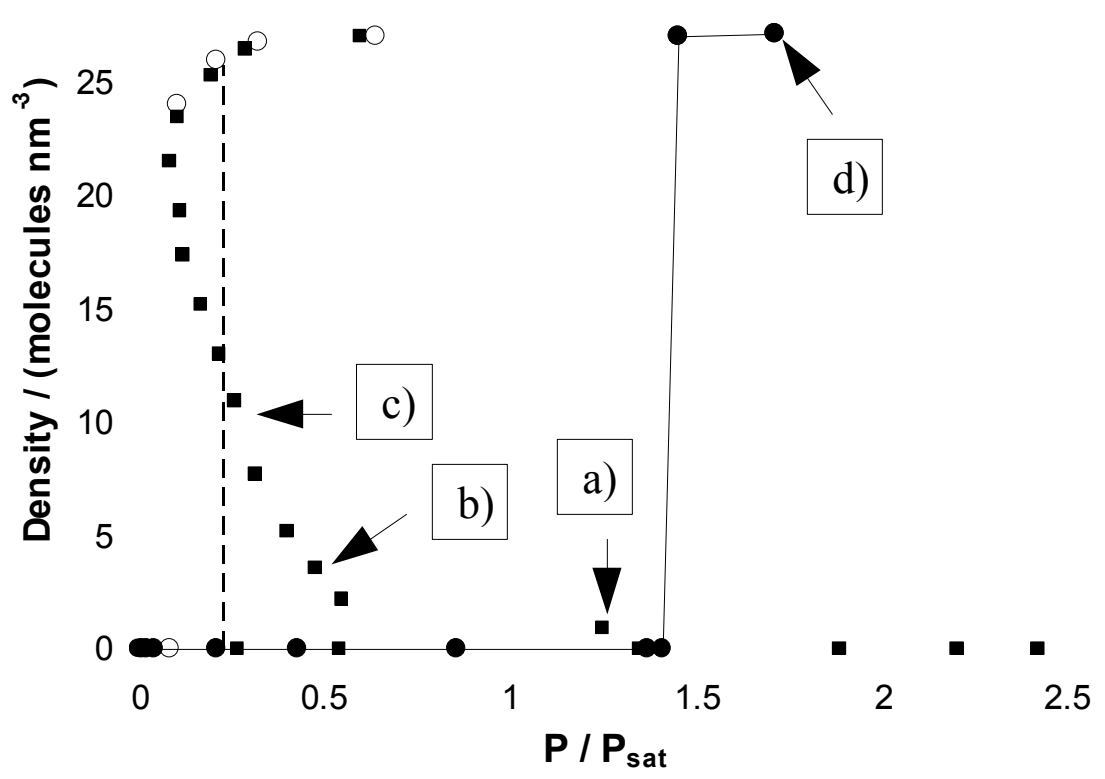

figure 9: Isotherm for water in a pore of width $1.64 \mathrm{~nm}$ at $293 \mathrm{~K}$. Symbols are the same as in figure 3. The small arrows and the labels mark the points at which density profiles and snapshot configurations were obtained. 

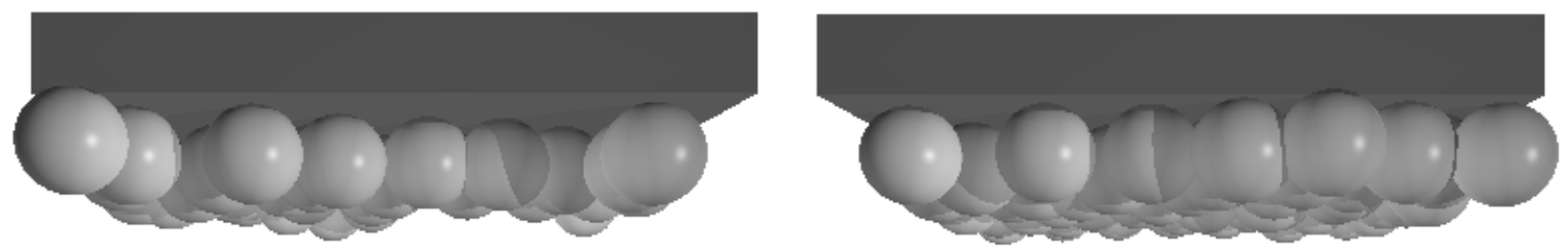

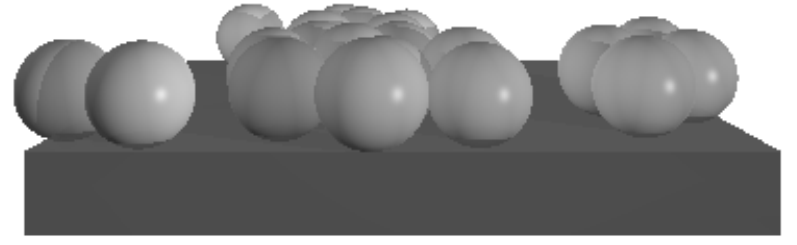

a)

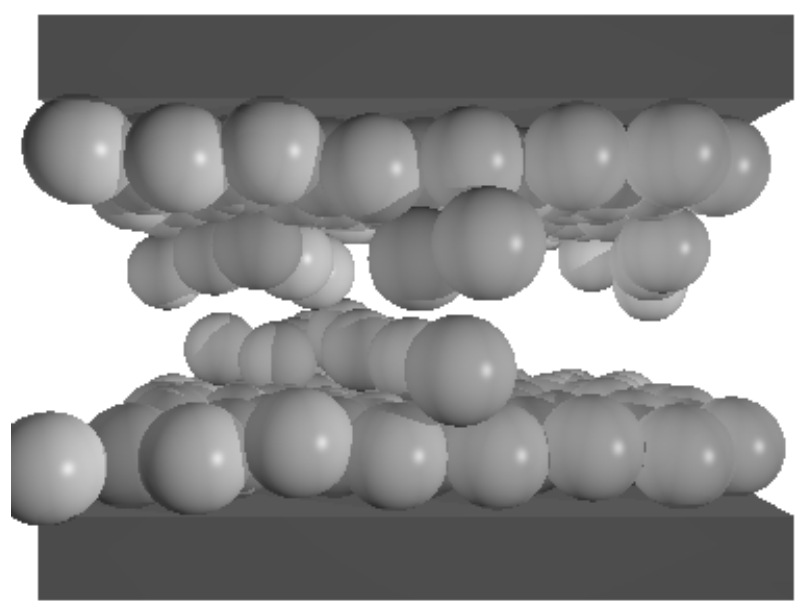

c)

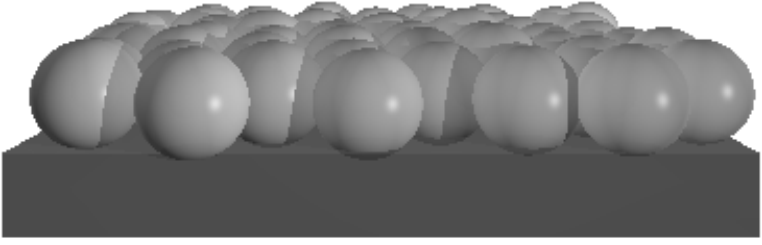

b)

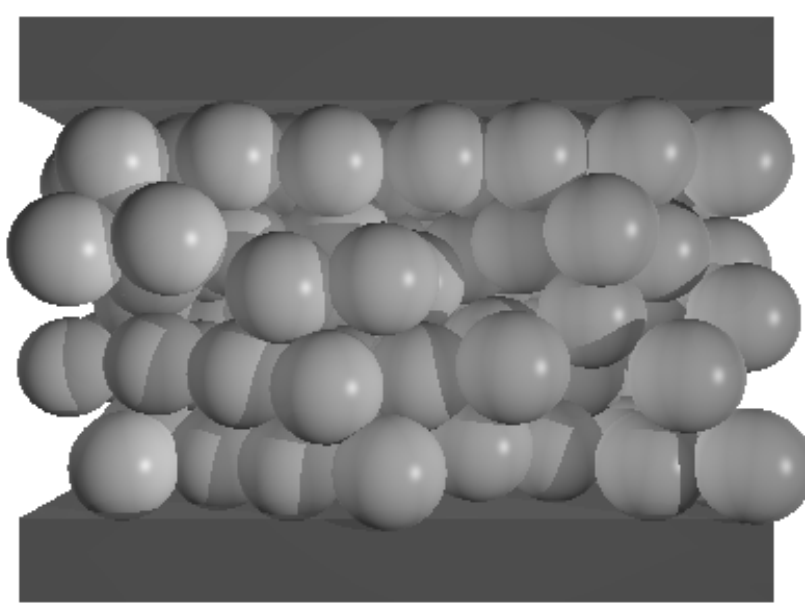

d)

figure 10: Snapshots of typical configurations corresponding to the points marked in figure 8 and the density profiles in figure 11. Grey spheres represent methane molecules. 


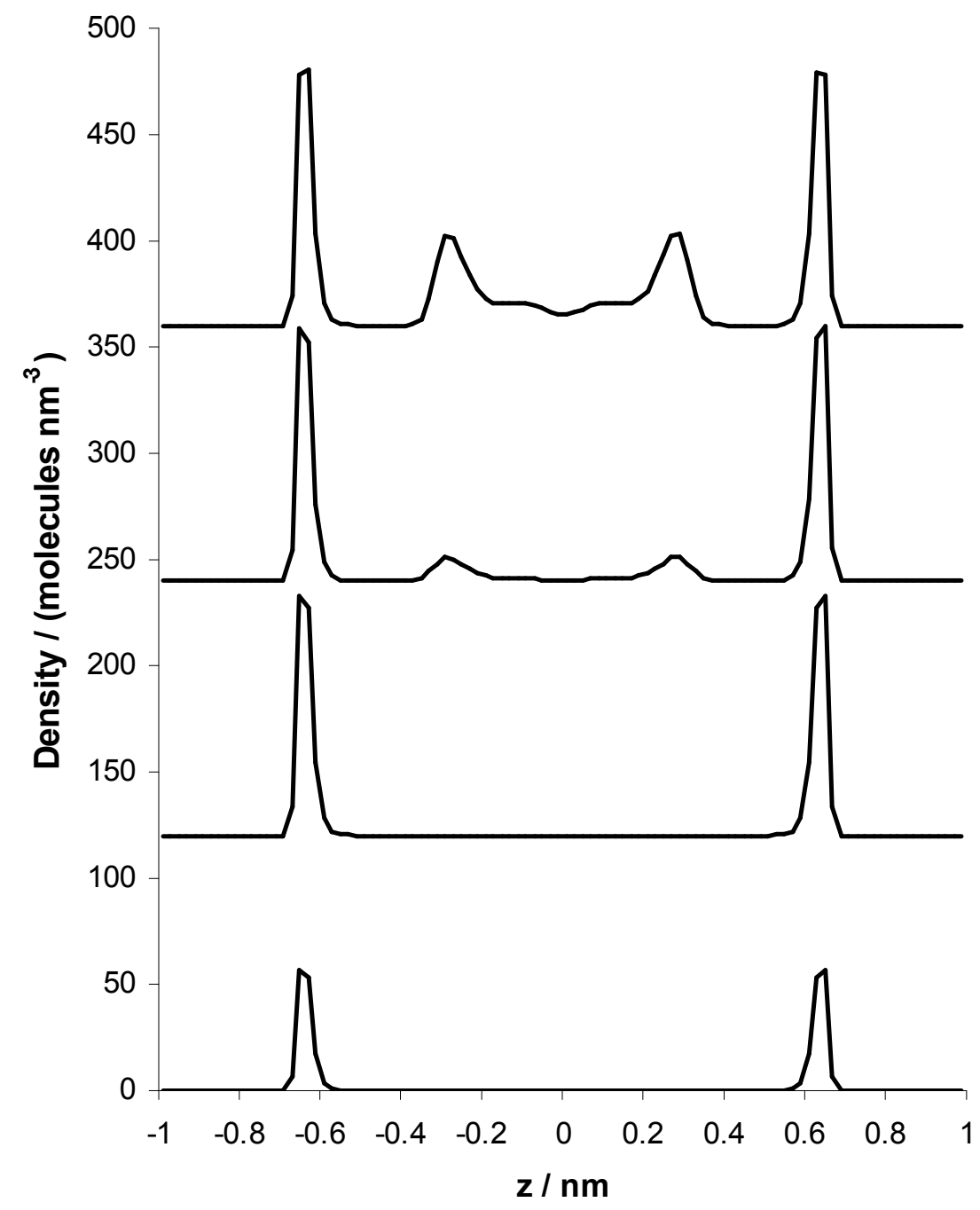

figure 11: Density profiles for methane adsorption at $86 \mathrm{~K}$ inside a $2 \mathrm{~nm}$ pore, from gauge-cell simulations. For ease of visualisation, each profile is shifted upwards by 120 molecules/nm3 relative to the preceding one. The average density values are, from bottom to top, 2.78 molecules/nm3 (corresponding to point a in figure 8 ), 5.56 molecules/nm3 (point b), 7.22 molecules/nm3 (point c) and 12.2 molecules/nm3 (point

d). 


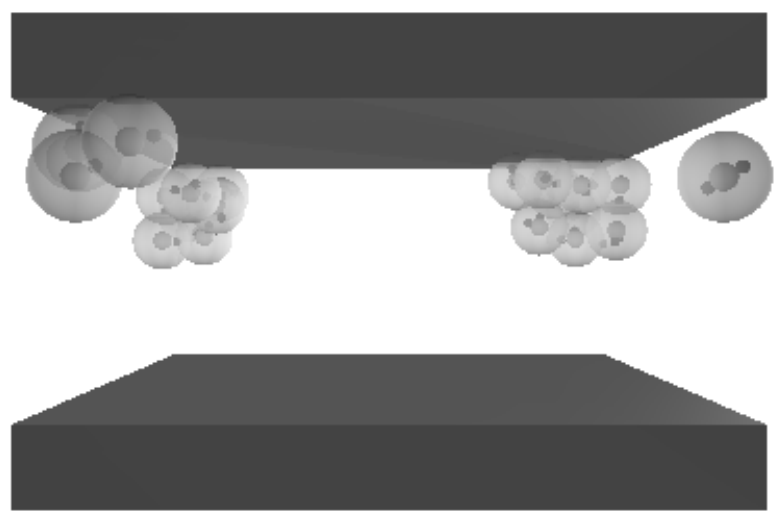

a)

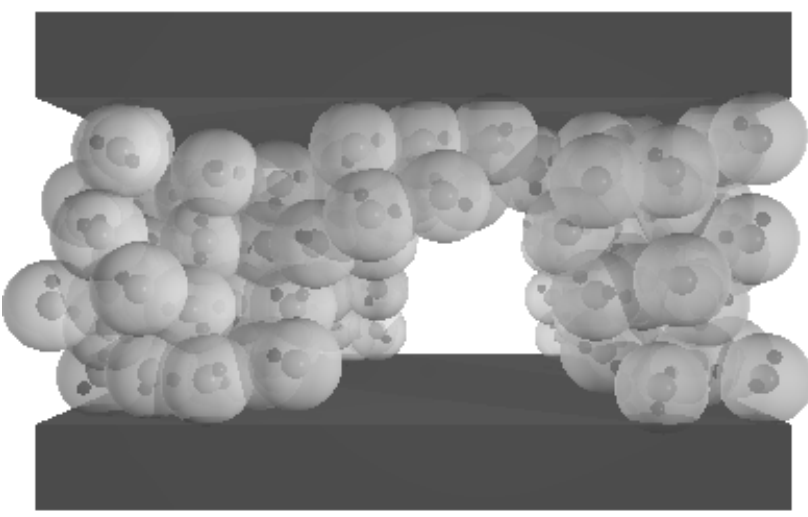

c)

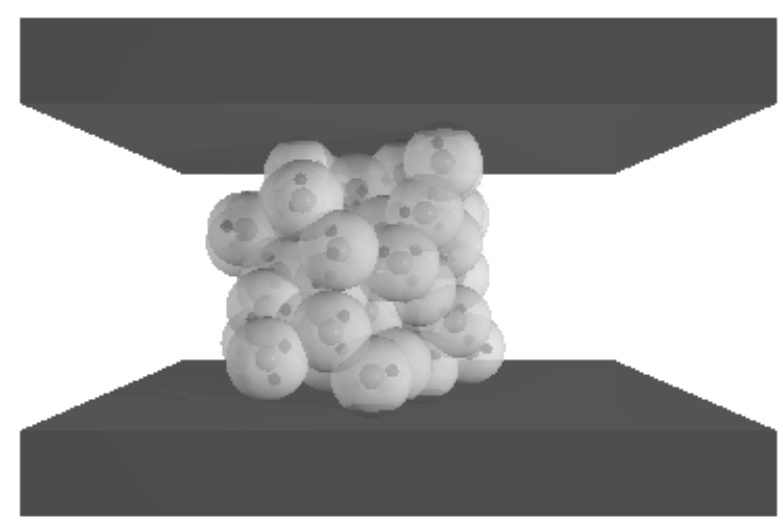

b)

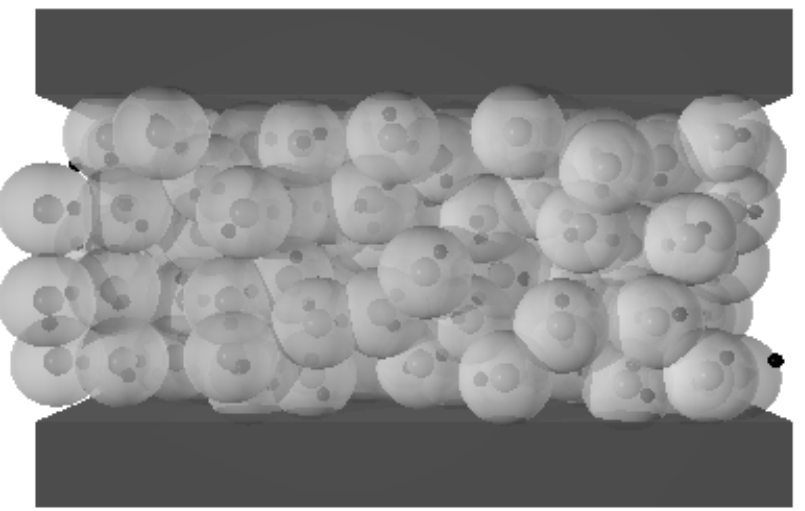

d)

figure 12: Snapshots of typical configurations corresponding to the points marked in figure 9 and the density profiles in figure 13 . The light grey transparent spheres represent water molecules. The solid spheres show the positions of the point charges within each water molecule, dark grey for negative charges and black for positive charges. 


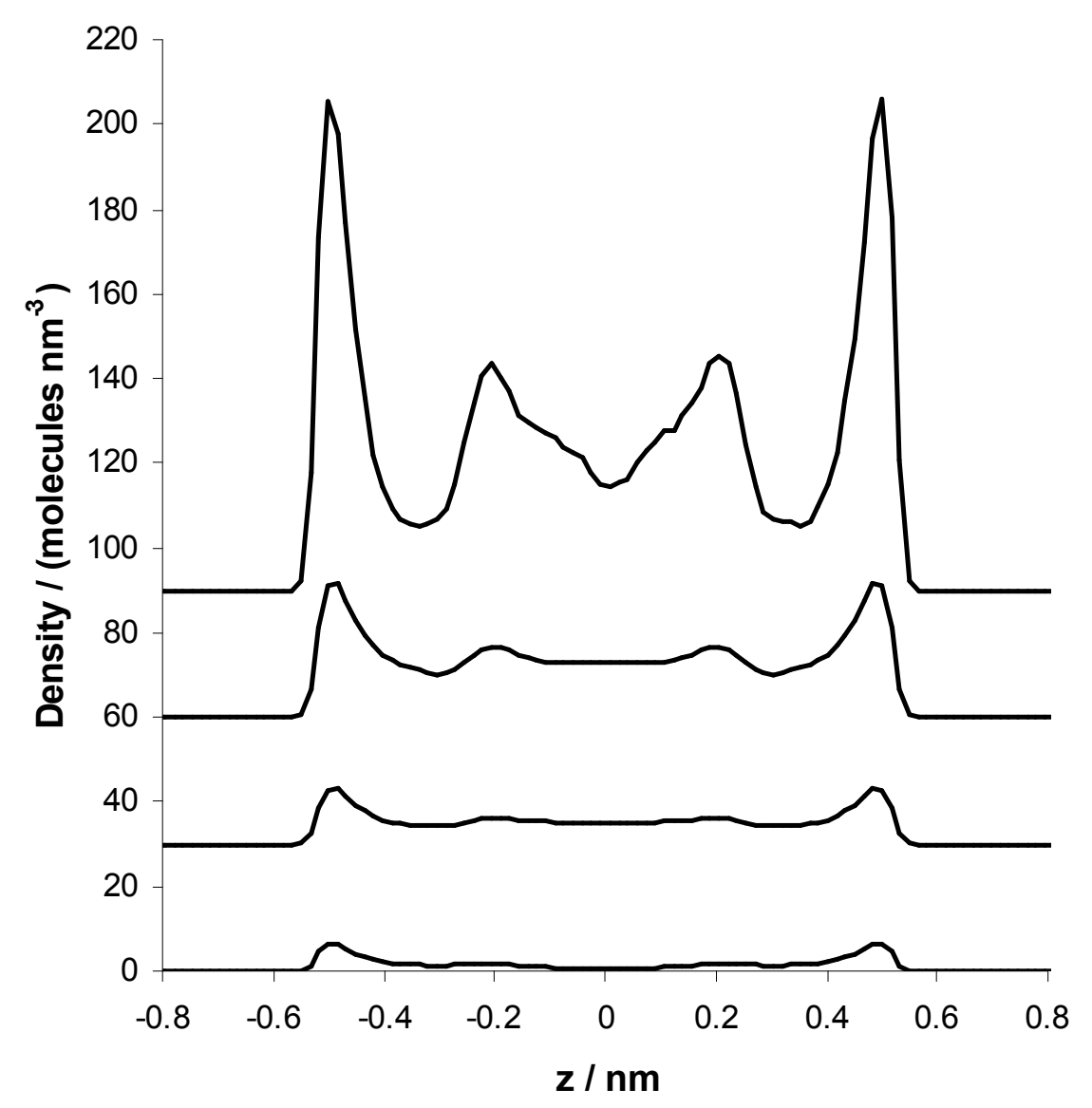

figure 13: Density profiles for water adsorption at $293 \mathrm{~K}$ inside a $1.64 \mathrm{~nm}$ pore, from gauge-cell simulations. For ease of visualisation, each profile is shifted upwards by 30 molecules/nm3 relative to the preceding one. The average density values are, from bottom to top, 1.36 molecules/nm3 (corresponding to point a in figure 9), 4.07 molecules/nm3 (point b), 10.16 molecules/nm3 (point c) and 27.1 molecules/nm3 (point

d). 


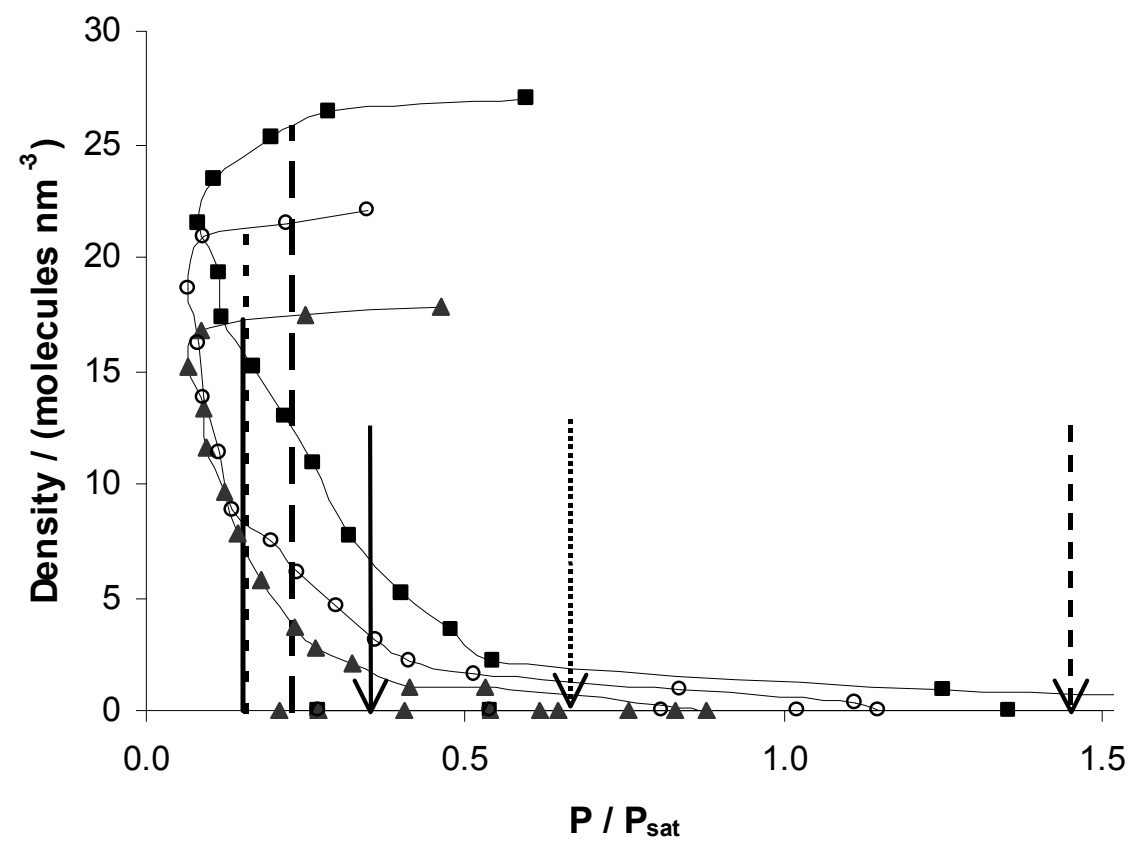

figure 14: Isotherms for water at $293 \mathrm{~K}$ adsorbed in pores of different width. Symbols are the results of the gauge-cell method: filled squares, $\mathrm{w}=1.64 \mathrm{~nm}$; open circles, $\mathrm{w}=$ $0.94 \mathrm{~nm}$; filled triangles $\mathrm{w}=0.64 \mathrm{~nm}$. The error bars are not shown to ease visualisation. Thin lines are a guide to the eye. Vertical lines are the values of Peq obtained by thermodynamic integration, while the vertical arrows show the values for Psc obtained from GCMC simulations: dashed line, $\mathrm{w}=1.64 \mathrm{~nm}$; dotted line, $\mathrm{w}=$ $0.94 \mathrm{~nm}$; solid line $\mathrm{w}=0.64 \mathrm{~nm}$. 